\title{
CARACTERÍSTICAS HABITACIONALES ENJUJUY, ARGENTINA, EN LA PRIMERA MITAD DEL SIGLO XX
}

\author{
Marcelo Jerez \\ Universidad Nacional de Jujuy, Jujuy, Argentina <marcelojerez@arnet.com.ar>
}

Resumen. Este artículo analiza las condiciones habitacionales de la población de la provincia de Jujuy en la primera mitad del siglo $\mathrm{xx}$, a la luz de los cambios $\mathrm{y}$ continuidades en el proceso de crecimiento económico local y nacional. Mucho se sabe acerca del problema de la vivienda en Argentina, sobre todo en el ámbito rioplatense, pero poco se ha profundizado sobre sus características e implicancias en otras regiones tales como en el Noroeste. De este modo, al centrar la atención en Jujuy se rescata no sólo la experiencia jujeña, sino también se develan ciertos rasgos habitacionales compartidos en esta región. A lo largo de este estudio se brindan nuevos aportes que son articulados dentro de aquel conocimiento en el nivel nacional y, cuando ello es posible, también discutidos.

Palabras clave: Condiciones materiales, vivienda, Jujuy.

Abstract. This paper examines the housing situation of the population of the province of Jujuy in the first half of the twentieth century, in light of the changes and continuance in the process of local economic growth. Much is known about the problem of housing in Argentina, especially in the River Plate area, but little has been deepened on its characteristics and implications in other regions such as the Northwest. Thus, the focus of the observation in Jujuy not only is rescued this provincial experience but it has also revealed certain housing traits shared in this region. In this study it is provided new contributions that are articulated with the national knowledge and, when possible, also discussed.

Key words: Material conditions, housing, Jujuy.

Fecha de recepción: diciembre de 2010. Fecha de aceptación: mayo de 2011

Am. Lat. Hist. Econ., año 19, núm. 2 (38), mayo-agosto, 2012, pp. 163-191 
$\mathrm{E}$ n una obra ya clásica, Alain Rouquié afirmaba que las condiciones de alojamiento en América Latina a principios del siglo XX eran en general deplorables. ${ }^{1}$ Esta descripción no se alejaba mucho de la realidad que, por entonces, exhibía la república Argentina, dada su notable evolución demográfica y urbana, lo que muy pronto trajo aparejado un patente desajuste entre el número de habitantes y la oferta de viviendas. En el presente trabajo histórico desplazamos nuestro lente de observación hacia el Noroeste argentino $^{2}$ y nos concentramos en la provincia de Jujuy, donde analizaremos las condiciones habitacionales de su población en la primera mitad del siglo XX.

Durante este periodo fue notoria la evolución demográfica de Jujuy producto, en buena medida, del crecimiento natural de sus habitantes, así como del arribo de una mayor inmigración, tanto extranjera como proveniente de otras provincias. Actividades como la minería, el comercio y principalmente la producción azucarera llevaron a la concentración demográfica en localidades que se constituyeron en polos de atracción. ${ }^{3}$ A principios de la centuria, pese a que Jujuy presentaba una población eminentemente rural, constituía una de las provincias del Noroeste con un importante ritmo de desarrollo urbano. ${ }^{4}$

Si bien la historiografía argentina ha abordado ampliamente el tema de la vivienda, por lo general se ha ocupado de las grandes ciudades de las provincias del Litoral rioplatense, en especial Buenos Aires. ${ }^{5}$ En consecuencia, muy poco se sabe acerca de lo acaecido en otros espacios alejados

${ }^{1}$ Rouquié, Extremo, 1991.

${ }^{2}$ Las provincias del Noroeste argentino están constituidas, en este estudio, por las provincias de Jujuy, Salta, Tucumán, Santiago del Estero, Catamarca y La Rioja. Para profundizar el análisis sobre ciertas características socioeconómicas propias de esta región, véanse, entre otros, Boleda, Población, 1993, y Bolsi, Problemas, 1997.

${ }^{3}$ Sala, "Redistribución", 2005.

${ }^{4}$ En efecto, entre 1914 y 1960, Jujuy, junto a Tucumán y Salta, son las que evidenciaban un elevado ritmo de crecimiento urbano, a diferencia de otras provincias con niveles más bajos como Catamarca, La Rioja o Santiago del Estero. Sin embargo, este crecimiento urbano tuvo niveles inferiores al experimentado en la región del Litoral. Esto resulta evidente al comparar entre 1914 y 1947 la evolución demográfica, por ejemplo, de la ciudad capital de Jujuy (7 622 a 31091 habitantes) o la de Tucumán (92 284 a 194000 habitantes) con la de la ciudad de Buenos Aires (1 575814 a 4554000 habitantes). Bolsi, Problemas, 1997.

${ }^{5}$ Son vastos los estudios sobre la cuestión de la vivienda que han subrayado el caso de Buenos Aires, entre ellos podemos citar a Yujnovsky, "Políticas", 1974; Korn y Torre, "Vivienda", 1985; Lecuona, Legislación, 1992; Torres, Mapa, 1993; Liernur, "Casas", 2000; Ballent, Huellas, 2005, y Aboy, Viviendas, 2005. Por su parte, no sólo son escasos los trabajos que examinan la temática de la vivienda en los espacios provinciales, sino que además no ahondaron demasiado en los rasgos habitacionales de los distritos del norte del país. Aquellos que contribuyeron a nuestra investigación, ciñeron su mirada en las provincias de Mendoza y Tucumán. Pero en esta última, el análisis se centra únicamente sobre la ciudad capital y sin profundizar en aspectos tales como los tipos de construcciones o niveles de hacinamiento y de inquilinos en otros centros urbanos. Cerdá, "Características", 2007-2008, y Natera, "Inquilinatos", 1996. 
del ámbito bonaerense, como es el caso de Jujuy. En este sentido, ciertos estudios centrados en la ciudad de Buenos Aires, provenientes del campo de la sociología y la demografía, han subrayado que $80 \%$ de quienes vivían en familia habrían adoptado, hacia 1936, el modelo nuclear -consistente en un matrimonio monogámico y sus hijos habitando una unidad habitacional independiente. ${ }^{6}$ Sin embargo, investigaciones recientes han matizado este fenómeno, sosteniendo que por estos años aún era muy común hallar familias que compartían su vivienda. ${ }^{7}$

La idea básica aquí defendida es que en Jujuy, como en varias provincias de la región, las viviendas presentaron ciertas características particulares que influyeron en la forma de habitar de su población, sobre todo en el ámbito urbano. Así, dada la magnitud de la insuficiencia habitacional habría sido habitual en su ciudad capital, San Salvador de Jujuy, la convivencia de grupos familiares en una sola vivienda. En este marco, surgen ciertas preguntas: ¿qué rasgos presentó la evolución demográfica y urbana de Jujuy a principios de siglo?, ¿qué características constructivas mostraban sus viviendas?, ¿'los niveles de hacinamiento e inquilinos eran importantes?, ¿qué particularidades exhibieron estos fenómenos en el contexto regional y nacional?, ¿todo ello incidió en la deficiencia habitacional de sus principales ciudades?, ¿cómo se expresó esta problemática en San Salvador de Jujuy?, y ¿qué matices presentó en esta urbe el ideal del modelo nuclear?

Con el propósito de responder estas interrogantes estructuramos nuestro estudio de acuerdo con tres núcleos temáticos: la evolución demográfica, los tipos de construcciones y los niveles de hacinamiento y de inquilinos registrados en Jujuy. Las fuentes trabajadas en esta investigación provienen fundamentalmente de los censos nacionales del periodo abordado ${ }^{8} \mathrm{y}$ se complementan con fuentes cualitativas procedentes de la prensa escrita de la época.

\footnotetext{
${ }^{6}$ Torrado, “Transición”, 2007, pp. 207-253. Gino Germani, por su parte, señala para aquel mismo año un porcentaje de $74 \%$ de las familias con hijos, sin contar las personas que vivían solas. Germani, Estructura, 1955, p. 51.

${ }^{7}$ Entre estos autores cabe destacar a Liernur, "Casas", 2000; Nari, Políticas, 2004; Ballent, "Perón”, 2004, y Aboy, “Arquitectura”, 2008.

${ }^{8}$ Somos conscientes de las dificultades que presenta este material, ya sea por la divergencia de los criterios adoptados o por algunas lagunas de información que obstaculizan la comparación de variables. De allí que en esta investigación, más que afirmaciones concluyentes, se examinan esencialmente indicios, se plantean descripciones sugestivas y desde luego también interrogantes. Las bases ideológicas de los censos, analizadas por Hernán Otero, nos han facilitado la lectura de estos registros estadísticos. Otero, "Demografía", 1999, y "Concepto", 2007, t. I.
} 


\section{EVOLUCIÓN DEMOGRÁFICA}

El primer rasgo que irrumpe al observar el conjunto del espacio jujeño es la diversidad que caracteriza a la provincia norteña en múltiples aspectos. Desde el punto de vista geográfico el territorio puede dividirse en cuatro grandes regiones internas: los valles centrales al sur; los valles subtropicales hacia el este; la Quebrada de Humahuaca, en la zona central, extendiéndose hacia el norte, y la Puna, abarcando los departamentos del oeste y del extremo norte. Estas dos últimas constituyen las llamadas tierras altas por estar ubicadas a una altura más elevada sobre el nivel del mar en relación con el resto. Otro rasgo distintivo estuvo dado por el origen indígena andino preponderante en la mayoría de la población de aquellas regiones. Por su parte las denominadas tierras bajas se hallan conformadas por los valles centrales, donde se encuentra el departamento y la ciudad capital y, en un predominante ambiente de selvas húmedas en las laderas de las montañas, los valles subtropicales, centro principal de la actividad azucarera en la provincia. ${ }^{9}$

En las primeras décadas del siglo xx la producción azucarera fue la mejor alternativa de articulación de las provincias del Noroeste argentino con la expansión económica, basada en la exportación de bienes primarios, que experimentaban las provincias del área pampeana. La expansión del cultivo de la caña de azúcar, sobre todo a partir de la segunda mitad de 1920, incidió en el crecimiento demográfico de Jujuy. Pero también contribuyeron a este fenómeno el desarrollo de la gran minería en la Puna -en el que participaron, además de firmas locales y extranjeras, empresarios azucareros- y de cierta producción agraria de importancia -como el tabaco- en los valles centrales. Todo ello alentó el crecimiento de los principales centros urbanos y de las actividades terciarias -como el comercio o el empleo estatal- desarrolladas en su interior. ${ }^{10}$

Sin duda uno de los factores que agudizó los efectos de la falta de viviendas fue el importante crecimiento poblacional que experimentaba Argentina hacia mediados de siglo. ${ }^{11}$ En este marco nacional, el fenómeno en Jujuy presentó características distintivas. En efecto, la provincia hacia

${ }^{9}$ A su vez la provincia de Jujuy se halla integrada por los siguientes departamentos: Capital, San Antonio y El Carmen (en los valles centrales); Ledesma, San Pedro, Valle Grande y Santa Bárbara (en los valles subtropicales); Humahuaca, Tilcara y Tumbaya (en la Quebrada); Cochinoca, Rinconada, Yavi, Susques y Santa Catalina (en la Puna). Teruel, "Panorama", 2006.

${ }^{10}$ La relevancia de estas actividades económicas hizo posible observar, en las primeras décadas del siglo, el inicio de un proceso de redistribución espacial de la población orientada, principalmente, hacia las zonas de los valles subtropicales, los valles centrales y la Puna. Sala, "Redistribución”, 2005.

${ }^{11}$ La magnitud de la evolución demográfica de Argentina, en este periodo, queda patente al apreciar los resultados de los censos nacionales de 1895 (4 044911 habitantes), 1914 (7 903662 
1947, en oposición a lo que sucedía con el país en su conjunto, presentaba una población predominantemente rural, rasgo, a la vez, común por entonces en el Noroeste. Pero dentro de este espacio regional, como ya se ha dicho, Jujuy era una de las provincias con un alto ritmo de desarrollo urbano. El crecimiento natural de sus habitantes y la mayor inmigración proveniente principalmente de provincias vecinas y de países limítrofes, fueron otros datos importantes del periodo. ${ }^{12}$

Los datos de los censos nacionales registrados entre 1895 y 1947 muestran el notable crecimiento demográfico de Jujuy. De los casi 50000 habitantes registrados a fines del siglo XIX, en 1914 suman 77 511, cifra que asciende hacia mediados de 1940 a 166700 (véase cuadro 1 ).$^{13} \mathrm{El} \mathrm{ritmo} \mathrm{de}$ crecimiento de esta provincia fue incluso mayor, en términos porcentuales, al que presentaron Salta y Tucumán en aquellos años. Este fenómeno se repite, y se acentúa aún más al comparar la evolución demográfica de San Salvador de Jujuy con las ciudades capitales de aquellas provincias.

Dentro de la provincia de Jujuy, los mayores aumentos demográficos se dieron en los departamentos Capital, Ledesma, San Pedro y Yavi (véase cuadro 2). De todos ellos, el caso que más sobresale es el incremento de la capital, que pasa de poco más de 14000 habitantes en 1914 a casi 42000 en 1947. Estos guarismos matizan de alguna forma la imagen tradicional de la zona azucarera como casi exclusiva concentradora de población en la provincia. ${ }^{14}$ En ese sentido, hacia aquel último año el número de habitantes del departamento Capital, por sí solo, no se aleja demasiado del que suman los departamentos azucareros de Ledesma y San Pedro.

Estos datos reflejan el constante e intenso crecimiento de las principales ciudades en Jujuy durante este periodo. Ciertamente, esto puede patentarse al comparar su evolución demográfica a lo largo de los primeros censos nacionales. En este proceso resalta, hacia 1947, el notorio incremento de tres urbes, ubicadas en la región de los valles centrales, subtropicales y la Puna: San Salvador de Jujuy, San Pedro y La Quiaca, respectivamente. Estas ciudades, precisamente, se encontraban ubicadas en tres de aquellos departamentos más poblados de la provincia (véase cuadro 3).

Pero al estudiar cada una de estas tres ciudades podemos observar un ostensible desequilibrio en su evolución. Todas ellas muestran un abrupto crecimiento hacia mediados de los años cuarenta, principalmente La

\footnotetext{
habitantes) y 1947 (15 893827 habitantes). Archivo General de la Nación (en adelante AGN), Censo general de la nación, 1947, y Censo de población, 1951, t. I.

${ }^{12}$ Ortiz y Caillou, "Primera", 1997.

${ }^{13}$ Dirección Provincial de Estadísticas y Censos (en adelante DiPEC), Censo 1991, Para darnos cuenta, 1991.

${ }^{14}$ Más allá del real y relevante foco de atracción laboral que durante este periodo constituyó el área azucarera (integrada por los departamentos Ledesma y San Pedro).
} 
CUADRO 1. EVOLUCIÓN DE LA POBLACIÓN DE LAS PROVINCIAS DE JUJUY, SALTA Y TUCUMÁN ENTRE 1914 Y 1947

\begin{tabular}{lrrc}
\hline \multicolumn{1}{c}{ Provincia } & 1914 & 1947 & $\begin{array}{c}\text { Crecimiento } \\
\text { (porcentaje) }\end{array}$ \\
Jujuy & 77511 & 166700 & 115.1 \\
Salta & 142156 & 290826 & 104.5 \\
Tucumán & 332933 & 593371 & 78.2 \\
& & & \\
Ciudad capital de provincia & & & \\
San Salvador de Jujuy & 7622 & 31091 & 307.9 \\
Salta & 28436 & 67403 & 137.0 \\
San Miguel de Tucumán & 93768 & 194166 & 107.0 \\
\hline
\end{tabular}

Fuente: elaboración propia con base en los censos nacionales de población de 1914 y 1947.

Quiaca, que en 1914 no contaba siquiera con los 2000 habitantes que requería el censo para otorgarle la categoría de ciudad. San Salvador y San Pedro, en cambio, exhibían un constante incremento desde principios del siglo pasado, aunque, mientras en el periodo 1914-1947 San Pedro creció aproximadamente 198\%, la ciudad capital lo hizo en más de 300\%. Indudablemente San Salvador era la ciudad más poblada y la que más había crecido en aquel lapso (véase gráfica 1). Esta evidente desigualdad a favor de la urbe capitalina queda reflejada hacia 1947, cuando reúne a más de $50 \%$ de la población urbana de la provincia y $74 \%$ de los habitantes de su departamento, el más poblado de Jujuy. ${ }^{15}$

En suma, estos datos reflejan un relevante movimiento demográfico en Jujuy hacia la mitad del siglo XX, siendo evidente el notable incremento poblacional del departamento Capital, donde su proceso de desarrollo urbano fue además el más importante de la provincia. En su principal ciudad, San Salvador de Jujuy, es muy probable que comenzaran a manifestarse ciertas dificultades habitacionales que por entonces experimentaban otros centros urbanos del país (tales como la escasez de viviendas, la multiplicación de los inquilinatos y el problema del hacinamiento). Seguidamente, profundizaremos sobre este supuesto, centrando nuestra atención en variables funcionales a este propósito; los tipos de construcciones y los niveles de hacinamiento y de inquilinos registrados en Jujuy.

\footnotetext{
${ }^{15}$ Mientras las ciudades de San Pedro y La Quiaca agrupaban 26 y 52\% de la población de sus departamentos. AGN, Censo general de la nación, 1947, y Censo de población, 1951, t. I.
} 
Am. Lat. Hist. Econ., año 19, núm. 2 (38), mayo-agosto, 2012, pp. 163-191

\section{CUADRO 2. EVOLUCIÓN INTERCENSAL DE LA POBLACIÓN DE LA PROVINCIA DE JUJUY SEGÚN REGIONES Y DEPARTAMENTOS, 1895-1947}

\begin{tabular}{llrrr}
\hline Región & Departamentos & 1895 & 1914 & 1947 \\
Valles centrales & Capital & 10165 & 14117 & 41955 \\
& El Carmen & 5406 & 7582 & 12632 \\
Valles subtropicales & San Antonio & 1049 & 1505 & 2245 \\
& Ledesma & 4786 & 12372 & 25724 \\
& San Pedro & 6451 & 15218 & 23579 \\
& Valle Grande & 1631 & 1721 & 2130 \\
Quebrada de & Santa Bárbara & s. d. & 1570 & 6736 \\
Humahuaca & Humahuaca & 4298 & 4262 & 11471 \\
& Tumbaya & 2030 & 2799 & 4479 \\
Puna & Tilcara & 2742 & 2886 & 6053 \\
& Santa Catalina & 2454 & 2510 & 3298 \\
& Cochinoca & 3741 & 4257 & 6913 \\
& Rinconada & 1681 & 1616 & 4916 \\
Población total & Yavi & 3279 & 4216 & 13119 \\
& Susques & s. d. & 880 & 1450
\end{tabular}
y 1947.

Fuente: elaboración propia con base en los censos nacionales de población de 1895, 1914

CUADRO 3. EVOLUCIÓN INTERCENSAL DE LOS CENTROS URBANOS MÁS POBLADOS DE LA PROVINCIA DE JUJUY, 1869-1947

\begin{tabular}{lllrrrr}
\hline Región & Departamento & Centro urbano & 1869 & 1895 & 1914 & 1947 \\
$\begin{array}{l}\text { Valles centrales } \\
\text { Valles }\end{array}$ & Capital & S. S. de Jujuy & 3072 & 4159 & 7622 & 31091 \\
$\quad$ subtropicales & San Pedro & San Pedro & 472 & 1380 & 2046 & 6105 \\
Puna & Yavi & La Quiaca & s. d. & 172 & s. d. $^{\text {a }}$ & 6768 \\
\hline
\end{tabular}

a El censo de 1914 no brinda datos de poblados con menos de 2000 habitantes.

Fuente: elaboración propia con base en el censo nacional de población de 1947. 


\section{GRÁFICA 1. EVOLUCIÓN DE LA POBLACIÓN DE SAN SALVADOR DE JUJUY, SAN PEDRO Y LA QUIACA}



Fuente: elaboración propia con base en los censos nacionales de 1869, 1914 y 1947.

\section{TIPO DE CONSTRUCCIONES}

Según el censo de 1895, y de acuerdo con el pensamiento liberal y positivista de la época, el país mostraba alentadores índices en las construcciones "de material", es decir, basadas en la utilización de ladrillo o piedra en lugar de adobe. ${ }^{16}$ No obstante, este fenómeno favoreció sobre todo a

${ }^{16}$ De acuerdo con los censos abordados en este apartado, básicamente, las viviendas "de adobe" hacen referencia a aquellas cuyas paredes fueron levantadas con bloques fabricados a base de paja y barro (techados frecuentemente con cañas o paja). Las casas "de material", por su parte, designan a aquellas con paredes construidas con ladrillos o piedras (y según el censo de 1895 con techos de azotea, teja, hierro o zinc). Cabe señalar que el censo de 1947 definió este tipo de construcciones como "de mampostería" (discriminando en otros apartados el material de los pisos y los techos). Con el propósito de mostrar ciertas tendencias constructivas en la provincia, aclaramos que a estas últimas las agrupamos en nuestro trabajo bajo la categoría de viviendas "de material”. 
las provincias del Litoral, evidenciándose mucho menos en otras regiones, como el Noroeste. Así, en el caso de Jujuy, 84.8\% de las viviendas era de adobe. Estos datos se hallaban en consonancia con los que mostraba el resto de la región, pero el porcentaje en Jujuy (84.8\%) superaba al de las provincias de Salta (81\%) y Tucumán (73.9\%), aunque se hallaba aún por debajo de los que exhibían Santiago del Estero (86.6\%) y Catamarca $(87.2 \%)$. Estos altos índices eran comparables con los que exhibía la región cuyana, ${ }^{17}$ con una alta proporción de construcciones de adobe y, naturalmente, un retraso en el conjunto de viviendas de material (véase cuadro 4).

En las primeras décadas del siglo $\mathrm{xx}$, la provincia de Jujuy comienza a evidenciar un aumento de la construcción de casas de material y un descenso en aquellas levantadas con adobe. Contribuyeron a ello las mejoras realizadas en las vías de comunicación (la llegada del ferrocarril y la ampliación de las rutas nacionales) que hicieron posible un mejor aprovisionamiento de los materiales de construcción. También fue un factor importante la decidida acción, tanto estatal como privada, al estimular y optar por otras alternativas al adobe en la edificación de viviendas. Hacia 1947 había descendido el porcentaje de casas construidas con adobe (46.6\%), mientras las viviendas de material aumentaron (19.8\%) en relación con los datos registrados en 1895. Por otro lado, un notorio incremento se registró en las casas construidas con otros materiales, principalmente la madera (13.7 por ciento)..$^{18}$

Estas cifras muestran, en primer lugar, que el aumento de las viviendas de material en Jujuy fue un proceso paulatino pero continuo desde principios de siglo, aunque el uso del adobe para mediados de 1940 seguía siendo importante en las construcciones de la provincia y la región (con la notable excepción de Tucumán). En segundo lugar evidencia cómo la utilización de otros materiales para construir viviendas, especialmente la madera, constituyó en estos años una práctica que comenzaba a ser difundida. La región del Litoral era nuevamente la que más había avanzado en el crecimiento de las viviendas de material, el otro extremo era Cuyo, donde se registraban menos cambios en este sentido. Estos datos, en términos regionales, patentizan una vez más, como bien señala Cerdá, qué zonas del país se habrían beneficiado más con el "progreso" experimentado durante la vigencia del modelo agroexportador a lo largo del periodo intercensal de 1895-1947 (véase cuadro 5). ${ }^{19}$

\footnotetext{
${ }^{17}$ Cuyo hace referencia en este estudio a la región geográfica que abarca a las provincias situadas en el centro oeste de la república argentina.

${ }^{18}$ AGN, Censo general de la nación, 1947, y Censo de vivienda, 1951, t. IV.

${ }^{19}$ Cerdá, "Características", 2007-2008.
} 
CUADRO 4. MATERIAL PREDOMINANTE EN LA CONSTRUCCIÓN DE VIVIENDAS, 1895 (PORCENTAJES)

\begin{tabular}{lrcc}
\hline Provincia & Material $^{\mathrm{a}}$ & Adobe & Otros $^{\text {materiales }}{ }^{\mathrm{b}}$ \\
Jujuy & & & \\
Salta & 13.9 & 84.8 & 1.3 \\
Tucumán & 18.3 & 81.0 & 0.7 \\
Santiago del Estero & 24.1 & 73.9 & 2.1 \\
Capital Federal & 7.9 & 86.6 & 5.5 \\
Buenos Aires & 93.8 & 0.3 & 6.0 \\
Santa Fe & 63.8 & 27.5 & 8.8 \\
Córdoba & 64.5 & 30.5 & 4.9 \\
Mendoza & 27.1 & 70.6 & 2.3 \\
San Juan & 15.0 & 83.4 & 1.6 \\
La Rioja & 5.9 & 94.0 & 0.1 \\
Catamarca & 15.1 & 83.7 & 1.3 \\
San Luis & 8.4 & 87.2 & 4.4 \\
Entre Ríos & 10.7 & 87.6 & 1.6 \\
Corrientes & 42.7 & 50.8 & 6.5 \\
\hline
\end{tabular}

${ }^{\text {a }}$ Casas construidas de piedra o ladrillo con techo de teja, zinc y azotea.

' Casas construidas de madera y "de fierro".

Fuente: elaboración propia con base en el censo nacional de población de 1895.

En Jujuy la mayoría de las casas de adobe se hallaba en el área rural, mientras que el crecimiento de las viviendas de material se evidenciaba en mayor medida en el ámbito urbano. En estos espacios la incipiente labor de la municipalidad cumplirá un papel importante. En efecto, como sucedía en otras partes del país, el crecimiento de las principales ciudades trajo aparejada la tarea de regular sus edificaciones. ${ }^{20}$ Este fue el caso del Reglamento General de Construcciones creado en 1921 y puesto en vigencia al año siguiente por la municipalidad de la ciudad más importante de la provincia: San Salvador de Jujuy. ${ }^{21}$ Este reglamento fomentaba el uso de material por sobre el adobe o el barro en la construcción de las viviendas, utilizando en ocasiones incentivos tales como la exención de impuestos comunales. También imponía la dirección y supervisión de las obras por

${ }^{20}$ Rigotti, "Ciudad”, 2000, t. VI.

${ }^{21}$ Este reglamento de construcción fue uno de los primeros en utilizarse en una ciudad argentina a principios del siglo XX, así, por ejemplo, Mendoza lo tendrá en 1927 y Mar del Plata en 1929. Ibid. 
Am. Lat. Hist. Econ., año 19, núm. 2 (38), mayo-agosto, 2012, pp. 163-191

\section{CUADRO 5. MATERIAL PREDOMINANTE EN LA CONSTRUCCIÓN DE VIVIENDAS, 1947 (PORCENTAJES)}

\begin{tabular}{lrrrrr} 
Provincia & Material & Madera & Adobe & Otros & Sin especificar \\
& & & & & \\
Jujuy & 19.8 & 13.7 & 46.6 & 13.5 & 6.4 \\
Salta & 23.8 & 18.0 & 39.0 & 13.8 & 5.4 \\
Tucumán & 56.1 & 13.6 & 7.7 & 19.7 & 2.9 \\
Santiago del Estero & 32.4 & 16.6 & 25.8 & 21.3 & 3.9 \\
Capital Federal & 94.8 & 3.7 & 0.0 & 0.1 & 1.4 \\
Buenos Aires & 76.7 & 7.8 & 8.7 & 5.6 & 1.2 \\
Santa Fe & 80.8 & 5.6 & 6.0 & 5.7 & 1.9 \\
Córdoba & 79.4 & 0.7 & 14.9 & 4.2 & 0.8 \\
Mendoza & 15.2 & 0.8 & 78.2 & 4.4 & 1.4 \\
San Juan & 9.4 & 1.9 & 75.2 & 12.4 & 1.1 \\
La Rioja & 17.3 & 1.3 & 63.4 & 14.9 & 3.1 \\
Catamarca & 13.9 & 2.2 & 64.9 & 16.8 & 2.2 \\
San Luis & 41.3 & 1.7 & 36.2 & 18.6 & 2.2 \\
Entre Ríos & 54.7 & 6.0 & 16.3 & 21.0 & 2.0 \\
Corrientes & 32.8 & 15.5 & 17.3 & 30.5 & 3.9 \\
\hline
\end{tabular}

Fuente: elaboración propia con base en el censo nacional de población de 1947.

parte de un ingeniero, arquitecto o constructor que pudiese asegurar la pervivencia del inmueble. ${ }^{22}$

Mientras estas iniciativas se tomaban en San Salvador de Jujuy, en otras urbes como San Pedro o La Quiaca las edificaciones parecen haber seguido otras pautas. Los datos del censo de 1947 revelan cómo en cada uno de los departamentos donde se ubicaban las ciudades mencionadas, los materiales de construcción utilizados en la mayoría de las casas eran diferentes. El departamento Capital contaba con el mayor porcentaje de viviendas construidas de material en la provincia (40.7\%). El de San Pedro, en cambio, exhibía una mayor presencia de casas levantadas con madera (36.5\%), seguida inmediatamente por aquellas de material (34.7\%). En ambos espacios, las unidades edificadas con adobe habían descendido, entre 1895 y 1947 , de 53.8 a $32.5 \%$ en el primer departamento y mucho más abruptamente en el segundo, de 91.7 a 13.3 por ciento. ${ }^{23}$

${ }^{22}$ Archivo Histórico Municipal de San Salvador de Jujuy (en adelante AHMJ), Reglamento General de Construcciones para la municipalidad de Jujuy, confeccionado por el señor concejal ingeniero Mario Romano, en acta del 31 de diciembre de 1921.

${ }^{23}$ AGN, Censo general de la nación, 1947, y Censo de vivienda, 1951, t. IV. 
En San Pedro, así como en el resto de los valles subtropicales, la madera, por su abundancia en esta zona, se convirtió en el material que conjugaba lo económico y lo práctico en gran parte de sus edificaciones. $\mathrm{Su}$ uso constituyó para muchos una alternativa válida para la construcción de viviendas o de los llamados ranchos. Pero también la utilización de madera correspondió en buena medida al área rural, fundamentalmente en la zona de los ingenios, para dar alojamiento a los trabajadores más humildes del azúcar. ${ }^{24}$ Esta fue la única región de la provincia donde las edificaciones de adobe fueron superadas ampliamente por las casas de madera y, en menor medida, por las de material.

Respecto a La Quiaca debemos ampliar por un momento la mirada hacia toda la región de la Puna y la Quebrada de Humahuaca, donde hacia 1947 y en contraposición al oriente jujeño, se registraba gran parte de las construcciones de adobe. Como ocurría en general en las poblaciones andinas, desde antaño este material había demostrado sus ventajas económicas y prácticas frente a los rigores del clima. A ello se añadía el inicio de un decidido apoyo estatal al turismo que fomentaba las viviendas construidas con adobe en estas regiones, consideradas como parte armónica del paisaje, del clima y de la historia misma de la provincia. Así, para 1947 el adobe predominaba entre sus construcciones en un porcentaje que oscilaba entre 60 y $85 \%$ según el departamento, en cambio, las proporciones de viviendas de material eran mucho más bajas. ${ }^{25}$

En la mayor ciudad de la Puna, La Quiaca, las edificaciones basadas en la utilización de otros materiales diferentes al adobe estuvieron a cargo principalmente de la acción privada. De todas maneras, aquel material continuaba siendo preponderante. Hacia 1947, en el departamento de Yavi, donde se ubicaba aquella urbe, el porcentaje de casas de adobe había descendido (77\%) respecto a lo registrado en 1895 (100\%). Sin embargo, estos índices se mostraban aún claramente superiores sobre las viviendas de material $(3 \%)$ y aquellas levantadas con otros materiales $(8 \%)$ (véase gráfica 2$) \cdot{ }^{26}$

El crecimiento de las viviendas de material en la provincia de Jujuy fue un proceso gradual a lo largo de las primeras décadas del siglo XX. Sin duda los sacrificios económicos de aquellos que levantaban este tipo de unidades habitacionales eran grandes, sobre todo durante la década de 1930, periodo en el que la población sufría los efectos de la crisis económi-

${ }^{24}$ Este fue el caso, por ejemplo, de los barrios de casillas de madera para obreros levantados por los propietarios del ingenio La Esperanza en el departamento de San Pedro. Para el personal de mayor jerarquía, lógicamente, se levantaron casas más amplias y de material. Sierra e Iglesias, Tiempo, 1998.

${ }^{25}$ AGN, Censo general de la nación, 1947, y Censo de vivienda, 1951, t. IV.

${ }^{26}$ Ibid. 







ca mundial. En este marco, indudablemente, fue difícil afrontar aquellos gastos. De ahí puede explicarse, en buena medida, el problema que significó para muchos terminar completamente la casa propia.

Ciertamente, en 1947 la provincia mostraba uno de los índices más altos del país en viviendas con piso de tierra, en consonancia con las tendencias que mostraban el Noroeste y Cuyo. En aquel año, 67\% de las casas jujeñas tenía piso de tierra, 13\% de baldosas y mosaicos, $6 \%$ de madera y $14 \%$ de otros materiales. De acuerdo con estos datos, es dable suponer la precariedad de gran parte de las viviendas de Jujuy, incluso pese a que sus techos eran de zinc. En efecto, este material estaba presente en más de la mitad de las casas de la provincia (58\%). Esta proporción era la más alta de la región y una de las mayores del país, superada lógicamente por las provincias del Litoral, mientras en las provincias cuyanas predominaban los techos construidos de otros materiales (probablemente el "torteado"). ${ }^{27}$

En síntesis, en Jujuy la mayor parte de las viviendas edificadas principalmente de adobe, material y madera presentaban pisos de tierra y techos de zinc. Estas características, se concentraban sobre todo en las casas que poseían entre una y cuatro habitaciones. De hecho, la existencia de unidades con pocas piezas fue, hacia 1947, uno de los rasgos característicos de la provincia y nuevamente frecuente en gran parte de sus homónimas del Noroeste (véase gráfica 3 ). ${ }^{28}$

Los elevados porcentajes de viviendas con escasas habitaciones dejan ver cómo en el ámbito urbano las dificultades habitacionales, como el hacinamiento, probablemente no sólo las padecían los inquilinos, sino también varias familias propietarias. Pero no sólo factores económicos contribuyeron a esta situación; también factores culturales. En tal sentido, es muy posible que muchos se vieran obligados, por falta de recursos económicos, a residir en este tipo de unidades, mientras otros tantos acostumbraran hacerlo, como aquellas familias provenientes del ámbito rural..$^{29}$ De una u otra forma, todo ello impactó seguramente en las condiciones habitacionales de las principales ciudades jujeñas, sobre todo en San Salvador de Jujuy. A continuación profundizaremos esta afirmación analizando aquellos fenómenos que, durante este periodo, contribuían a hacer deficiente la vida de la población argentina: el hacinamiento y los inquilinatos.

${ }^{27} \mathrm{Ibid}$. Torteado es el nombre que se le da al techo construido con adobe, caña y paja, muy utilizado en gran parte de las casas de adobe en el Noroeste en aquellos años y muy posiblemente también en otras regiones como la de Cuyo.

${ }^{28}$ Ibid.

${ }^{29}$ Así por ejemplo, en la zona rural puneña el uso de pequeñas viviendas constituía una práctica de larga data. En estas residencias el hacinamiento de familias enteras fue un rasgo habitual de la región durante el periodo estudiado y, según Bolsi, a lo largo de la centuria. Bolsi, "Ruralia", 2005. 


\section{GRÁFICA 3. VIVIENDAS SEGÚN CANTIDAD DE PIEZAS EN LA PROVINCIA DE JUJUY, 1947}



Fuente: elaboración propia con base en el censo nacional de población de 1947.

\section{El hacinamiento}

Hacia 1943 se levanta en todo el país el IV Censo Escolar, del Analfabetismo y de la Vivienda. En Jujuy sus resultados pondrían en evidencia la dimensión que había asumido el problema del hacinamiento y su ubicación dentro del conjunto nacional. De acuerdo con el censo se utilizaron dos pautas de evaluación del hacinamiento: colectivo (más de cuatro familias que compartían una casa) e individual (más de cuatro miembros de una familia que dormían todos en una misma pieza)..$^{30}$ Según este relevamiento, el hacinamiento era un problema común en Argentina en aquellos años.

En Jujuy, el hacinamiento colectivo, aunque frecuente en la zona rural, no presentaba los porcentajes más elevados en el ámbito nacional. Para

${ }^{30} \mathrm{El}$ hacinamiento fue definido por el censo como: "[...] vida dentro de un medio cerrado, de un número excesivo de personas. En tal sentido, el hacinamiento puede revestir dos formas: 'hacinamiento colectivo', que el grupo comprenda a un determinado número de familias que viven todas en una misma casa; o 'hacinamiento individual', que se refiera a personas de una o más familias que duermen todas en la misma pieza. Concretando estos conceptos, diremos que hacinamiento colectivo es la convivencia en una misma casa de por lo menos cuatro o más familias. Cuando en una casa conviven dos o tres familias, que por lo general se hallan ligadas entre sí por vínculos de parentesco, el problema desde el punto de vista social se atenúa, razón por la cual lo llamamos entonces 'convivencia sin hacinamiento'." AGN, IV Censo Escolar de la Nación, 1948, t. I., pp. 159, 160. 
1943, 36.8\% de las familias jujeñas censadas compartía la vivienda con otras, de las cuales $71.8 \%$ estaba constituido por grupos de entre cuatro y seis familias y $23.8 \%$ de entre siete y diez familias por casa. En el plano regional estos porcentajes eran relativamente similares a los que exhibía el resto de las provincias del Noroeste.

A diferencia del hacinamiento colectivo, el hacinamiento individual era más recurrente e importante en la provincia y afectaba a $55.5 \%$ de las familias censadas. Debe tenerse en cuenta que la mayoría de estos casos traducía de hecho situaciones en que el núcleo familiar no solamente dormía, sino que también cocinaba, comía y hasta trabajaba en la misma habitación..$^{31}$ Estos índices eran comunes en el Noroeste -en Santiago del Estero el fenómeno se presentaba en $61 \%$ de los hogares- y elevados en relación con provincias de otras regiones como Mendoza o Córdoba. Cabe destacar que en la época el hacinamiento individual era calculado considerando cuatro personas por habitación. Esta relación en los hogares urbanos en pocas ocasiones coincidía con la media de los miembros de una familia, conformada en general con más integrantes, y mucho menos en los hogares rurales que contaban con amplios grupos familiares, como en la Puna por ejemplo (véase cuadro 6). ${ }^{32}$

En el interior de la provincia de Jujuy, los valles subtropicales presentaban el mayor porcentaje de hacinamiento individual (60.2\%). La Puna y la Quebrada mostraban cerca de $58 \%$ de casos, mientras que en la zona de los valles centrales la proporción ascendía a $49.1 \%{ }^{33}$ Estos datos resaltan la importancia que tenía en Jujuy el hacinamiento individual. Al centrar nuestro estudio en los departamentos Capital, San Pedro y Yavi, podemos constatar la gravedad de esta situación. En todos estos distritos, los porcentajes llegan a alrededor de 50\%. Es decir que, de las familias censadas, aproximadamente la mitad de las mismas, y en algunos casos muchas más, se hallaban residiendo en una única habitación (véase cuadro 7).

$\mathrm{Al}$ igual que con el hacinamiento colectivo, en Jujuy los mayores casos de hacinamiento individual se producían en la zona rural, aunque los índices también eran significativos en la zona urbana, llegando casi a 50\% del total registrado. En la mayoría de las ciudades, cerca de la mitad de las familias censadas padecía de hacinamiento individual. Estos datos revelan que, si bien eran notorias las diferentes condiciones de vida en general de 1944.

${ }^{31}$ AGN, Dirección de Investigaciones Económicas y Sociales, Intervención Federal de Jujuy,

${ }^{32}$ La población de la Puna, a mediados del siglo xx, se destacaba por su alto crecimiento natural, producto del declive de la mortalidad en un contexto de alta fecundidad. Bolsi, "Ruralia", 2005.

${ }^{33}$ AGN, Dirección de Investigaciones Económicas y Sociales, Intervención Federal de Jujuy, 1944. 
Am. Lat. Hist. Econ., año 19, núm. 2 (38), mayo-agosto, 2012, pp. 163-191

CUADRO 6. FAMILIAS EN UNA PIEZA

EN JUJUY, MENDOZA Y CÓRDOBA, 1943 (PORCENTAJES)

\begin{tabular}{lccccc}
\hline & $\begin{array}{c}\text { Familias con } \\
\text { hacinamiento } \\
\text { individual }\end{array}$ & $\begin{array}{c}\text { De 4 a } 5 \\
\text { personas en que conviven } \\
\text { Provincias }\end{array}$ & $\begin{array}{c}\text { De } 6 \text { a 8 } \\
\text { personas }\end{array}$ & $\begin{array}{c}\text { 9 y más } \\
\text { personas }\end{array}$ & Total \\
Jujuy & 55.5 & 58.7 & 35.8 & 5.6 & 100 \\
Mendoza & 31.7 & 66.8 & 28.3 & 5.0 & 100 \\
Córdoba & 34.1 & 64.7 & 29.8 & 5.4 & 100 \\
\hline
\end{tabular}

Fuente: elaboración propia con base en el IV Censo Escolar de la Nación de 1943.

CUADRO 7. FAMILIAS QUE PADECEN HACINAMIENTO INDIVIDUAL EN JUJUY POR DEPARTAMENTOS, 1943

(PORCENTAJES)

\begin{tabular}{lccccc}
\hline & \multicolumn{4}{c}{$\begin{array}{c}\text { Casos según cantidad } \\
\text { de personas convivientes }\end{array}$} \\
Departamento & $\begin{array}{c}\text { Porcentaje de } \\
\text { familias }\end{array}$ & De 4 a 5 & De 6 a 8 & 9 y más & Total \\
Jujuy Capital & 46.4 & 60.6 & 34.2 & 5.2 & 100 \\
El Carmen & 54.7 & 54.3 & 41.8 & 3.9 & 100 \\
San Antonio & 63.7 & 43.5 & 44.0 & 12.5 & 100 \\
Ledesma & 60.4 & 60.4 & 33.2 & 6.4 & 100 \\
San Pedro & 55.6 & 51.3 & 40.8 & 8.0 & 100 \\
Valle Grande & 67.9 & 52.3 & 40.4 & 7.3 & 100 \\
Humahuaca & 59.2 & 58.8 & 35.9 & 5.3 & 100 \\
Tilcara & 51.9 & 58.5 & 34.6 & 7.0 & 100 \\
Tumbaya & 64.0 & 55.0 & 37.5 & 7.5 & 100 \\
Yavi & 55.1 & 64.6 & 33.4 & 1.9 & 100 \\
Santa Catalina & 61.5 & 63.9 & 32.1 & 4.0 & 100 \\
Cochinoca & 60.5 & 58.6 & 34.5 & 6.8 & 100 \\
Rinconada & 60.2 & 57.5 & 39.3 & 3.2 & 100 \\
& & & & & \\
\hline
\end{tabular}

Fuente: elaboración propia con base en el IV Censo Escolar de la Nación de 1943. 
la población rural y urbana, las deficiencias habitacionales en la ciudad eran aún muy similares a las que padecían varias familias campesinas en la provincia (véase cuadro 8 ).

Analizando la magnitud que adquiría el problema en la principal ciudad de Jujuy, estos datos dan cuenta que en San Salvador de Jujuy 39.1\% de los habitantes desenvolvía su existencia en una sola pieza. Esta importancia del hacinamiento individual -al subrayar la convivencia de una familia en un único ambiente y teniendo en cuenta la preponderancia de las viviendas con pocas habitaciones- induciría, en un primer momento, a inferir el triunfo del ideal del modelo nuclear. No obstante, dada la significativa evolución demográfica y urbana de la provincia, es dable suponer que en esta ciudad el desfase entre el número de habitantes y el de viviendas contribuyera a que varias casas -aun cuando no contaran con múltiples cuartos- se encontraran ocupadas por más de un grupo familiar. En este sentido, para muchas de estas personas el alquiler se habría constituido en una práctica muy extendida por entonces. ${ }^{34}$

\section{Propietarios e inquilinos}

El censo de 1947 registró que en Jujuy sólo 30\% de las casas estaban ocupadas por sus dueños. Estos datos ubicaban a este distrito entre aquellos con mayor proporción de inquilinos no sólo de la región, sino del país, superado únicamente por la capital federal con un porcentaje de $18 \%$ de propietarios. Estas tendencias reflejaban, una vez más, lo difícil que era la obtención de la casa propia en Argentina y, dentro de este marco, el nivel de gravedad que asumía esta cuestión en Jujuy (véase cuadro 9).

Ciertos estudios han resaltado, centrando especialmente su observación en la ciudad de Buenos Aires, la incidencia que tuvieron el congelamiento de los alquileres y la prohibición de los desalojos en los bajos porcentajes de propietarios. ${ }^{35}$ Esto habría contribuido a que cada vez más personas optaran por el alquiler, por los costos que representaba, en detrimento de la construcción de la vivienda propia. No obstante, este razonamiento sin duda adquiere algunos matices si tenemos en cuenta las realidades particu-

${ }^{34}$ Esto hallaría correspondencia con un artículo periodístico que, en 1934, denunciaba: "Tenemos en el radio urbano de esta capital, numerosas casas de inquilinato [y] 'conventillos', verdaderas pocilgas que no ofrecen a las personas pobres que las habitan nada que pueda considerarse como una relativa comodidad, aparte de que se convierten aquellas, por el hacinamiento de sus moradores y por su estado lamentablemente antihigiénico, en una serie de focos infecciosos y hasta en antros de corrupción e inmoralidades.” Biblioteca Popular de Jujuy, sección Hemeroteca (en adelante BPJ-SH), La Opinión, 14 de noviembre de 1934, p. 3.

${ }_{35}$ Medida que se extiende desde 1943 hasta 1955. Torre y Pastoriza, "Democratización", 2002 , t. VIII. 
Am. Lat. Hist. Econ., año 19, núm. 2 (38), mayo-agosto, 2012, pp. 163-191

CUADRO 8. HACINAMIENTO INDIVIDUAL SEGÚN ÁREA URBANA Y RURAL EN JUJUY, 1943

\begin{tabular}{lccccc}
\hline \multirow{2}{*}{ Zona } & $\begin{array}{c}\text { Porcentaje } \\
\text { de familias }\end{array}$ & \multicolumn{4}{c}{ Casos según cantidad de personas } \\
& & De 4 a 5 & De 6 a 8 & 9 y más & Total \\
Urbana & 48.4 & 62.4 & 33.2 & 4.4 & 100 \\
Cuasi urbana & 57.4 & 61.8 & 33.1 & 5.1 & 100 \\
Rural & 57.9 & 56.5 & 37.4 & 6.1 & 100 \\
\hline
\end{tabular}

Fuente: elaboración propia con base en el IV Censo Escolar de la Nación de 1943.

CUADRO 9. PROPIETARIOS E INQUILINOS EN ARGENTINA, 1947 (PORCENTAJES)

\begin{tabular}{lcc}
\hline Jurisdicción & Propietarios & Inquilinos \\
Jujuy & 29.9 & 70.1 \\
Salta & 34.5 & 65.5 \\
Tucumán & 40.3 & 59.7 \\
Santiago del Estero & 50.4 & 49.6 \\
Capital Federal & 17.6 & 82.4 \\
Buenos Aires & 42.0 & 58.0 \\
Santa Fe & 39.5 & 60.5 \\
Córdoba & 41.9 & 58.1 \\
Mendoza & 32.2 & 67.8 \\
San Juan & 35.0 & 65.0 \\
La Rioja & 55.0 & 45.0 \\
Catamarca & 57.8 & 42.2 \\
San Luis & 41.8 & 58.2 \\
Entre Ríos & 53.6 & 46.4 \\
Corrientes & 51.4 & 48.6 \\
\hline
\end{tabular}

Fuente: elaboración propia con base en el censo nacional de población de 1947. 
lares que presentaban las distintas provincias argentinas a mediados del siglo pasado. En Jujuy, por ejemplo, al profundizar el análisis en su interior esta explicación se complejiza y adquiere algunos rasgos propios. De este modo, los bajos porcentajes de propietarios en Jujuy muestran tendencias distintivas en sus diferentes regiones (véase cuadro 10).

Conforme a estas estimaciones, los principales departamentos de la Quebrada de Humahuaca y de la Puna no exhiben, en general, los porcentajes de inquilinos más altos de la provincia, aunque debemos señalar que la participación del total de viviendas en ambas también fue menor en relación con el resto de las regiones. En los departamentos donde los índices eran elevados es muy posible que la cuestión estuviera vinculada con la existencia de ciertos terratenientes que poseían propiedades de extensiones considerables. Estos combinaban frecuentemente actividades comerciales con la función pública, y percibían una renta de los campesinos que históricamente allí residían, no sin ciertas arbitrariedades. ${ }^{36}$

Cabe señalar que tempranamente aquellos campesinos habían levantado sus voces contra los abusos en el cobro de los arriendos, solicitando una decidida intervención gubernamental que hiciese posible la restitución de estas tierras. Así, por mediación oficial, en la década de 1880 compraron algunas fincas (especialmente en Santa Catalina), ${ }^{37}$ pero la problemática en torno a la tierra continuó y adquirió relevancia en 1946, durante la presidencia de Perón, con el denominado Malón de La Paz, cuando los puneños se trasladaron a la Capital Federal para pedir la expropiación de los latifundios. En 1949, Perón dictó el solicitado decreto, transformando las propiedades expropiadas en tierras fiscales. ${ }^{38}$

Por su parte, los valles centrales y los subtropicales son las regiones con mayor proporción de inquilinos. Este fenómeno se agudizaba mucho más en aquella última, mostrando la menor presencia de propietarios de Jujuy. ${ }^{39}$ Pero el censo no discriminó estos datos para los ámbitos urbano y rural, con lo cual gran parte de los inquilinos registrados muy probablemente correspondió a los numerosos trabajadores del azúcar instalados en esta zona. Si bien los dueños de los ingenios construían viviendas para albergar a sus obreros, no se les entregaba su propiedad. Esto ayudaría a

${ }^{36}$ Fleitas y Teruel, "Política”, 2007.

${ }^{37}$ Teruel y Bovi, "Ordenamiento", 2010.

${ }^{38}$ Para ampliar acerca de esta temática véase Belli, Slavutsky y Rueda, Malón, 2007, y Kindgard, "Tradición", 2004.

${ }^{39}$ La excepción en los valles subtropicales, estuvo claramente marcada por el departamento Valle Grande. Su elevado porcentaje de propietarios (77\%), sin duda estuvo vinculado con la escasa población y viviendas censadas. Además, este distrito fue uno de los pocos donde el gobierno provincial había fraccionado y entregado en propiedad gran parte de las tierras a los campesinos, convirtiendo a la mayoría de ellos en propietarios. Teruel y Bovi, "Ordenamiento", 2010. 


\section{CUADRO 10. VIVIENDAS CLASIFICADAS \\ SEGÚN SITUACIÓN JURÍDICA DE SUS OCUPANTES EN JUJUY POR DEPARTAMENTOS, 1947}

\begin{tabular}{llrcr}
\hline $\begin{array}{l}\text { Regiones de } \\
\text { la provincia }\end{array}$ & Departamento & Total de viviendas & $\begin{array}{c}\text { Propietarios } \\
\text { (porcentajes) }\end{array}$ & $\begin{array}{c}\text { Inquilinos } \\
\text { (porcentajes) }\end{array}$ \\
Valles centrales & Jujuy Capital & 8491 & 26.9 & 73.1 \\
& El Carmen & 2371 & 27.4 & 72.6 \\
& San Antonio & 408 & 69.1 & 30.9 \\
Valles & Ledesma & 5105 & 8.2 & 91.8 \\
subtropicales & San Pedro & 5376 & 6.1 & 93.9 \\
& Valle Grande & 416 & 77.6 & 22.4 \\
Quebrada de & Santa Bárbara & 1542 & 11.7 & 88.3 \\
Humahuaca & Humahuaca & 2469 & 48.8 & 51.2 \\
& Tilcara & 1208 & 39.8 & 60.2 \\
Puna & Tumbaya & 829 & 41.5 & 58.5 \\
& Yavi & 2781 & 55.3 & 44.7 \\
& Santa Catalina & 713 & 89.8 & 10.2 \\
& Cochinoca & 1487 & 76.2 & 23.8 \\
& Susques & 279 & 93.2 & 6.8 \\
Totales & Rinconada & 1146 & 24.5 & 75.5 \\
\hline
\end{tabular}

Fuente: elaboración propia con base en el censo nacional de población de 1947.

comprender la baja proporción de propietarios existentes, sobre todo en los departamentos de Ledesma y San Pedro, donde se ubicaban los ingenios azucareros más importantes de la provincia.

Ante la ausencia de registros censales por ciudad, analizaremos los departamentos donde se hallaban los principales centros urbanos. En tal sentido, los distritos Capital y San Pedro, por sobre Yavi, muestran los índices más altos de inquilinos. De acuerdo con el crecimiento que evidenciaron sus ciudades, podemos deducir que este fenómeno repercutió en las mismas, aunque suponemos que en menor magnitud en San Pedro y La Quiaca. Los departamentos donde se ubicaban estas urbes presentaban una importante población rural. Precisamente a esta zona pertenecían tanto los puneños afectados por la cuestión de la tierra como los numerosos obreros del azúcar. En las ciudades de San Pedro y La Quiaca, en cam- 
bio, la mayor parte de aquellos que recurrían al alquiler se dedicaba muy probablemente a tareas terciarias y de servicios. ${ }^{40}$

La Capital, por su parte, no sólo exhibía bajos índices de propietarios, sino que, a diferencia de los anteriores distritos, su principal ciudad, San Salvador de Jujuy, reunía a más de $70 \%$ de la población del departamento. Por ello es lógico inferir que en esta urbe -más que en el resto- su elevado nivel de inquilinos llegó a constituirse en una cuestión ciertamente acuciante. Los que padecían esta situación pertenecían a un heterogéneo grupo de los sectores bajos y medios de la sociedad. Pero sin duda una parte importante estuvo integrada principalmente por estos últimos. Es decir, aquellos que habían podido afrontar los constantes vaivenes del valor de los alquileres durante las primeras décadas del siglo. Entre ellos, posiblemente se contaban muchos empleados públicos. Apoyamos esta afirmación en el análisis de los precios de los alquileres.

Según el censo de 1947, 62\% de las viviendas alquiladas pagaba una renta entre 76 y $100 \mathrm{~m} \$ \mathrm{n}$ por mes. Estos precios estaban lejos de las posibilidades de un jornalero que ganaba mensualmente alrededor de $60 \mathrm{~m} \$ \mathrm{n}$. Para el año de este relevamiento, el valor del alquiler insumía aproximadamente $66 \%$ del sueldo de un ordenanza y $30 \%$ de un empleado medio del sector público. Esto evidencia que los trabajadores estatales, principalmente de mediana y más alta jerarquía, se encontraban mejor posicionados para costear estos gastos.

Asimismo, si tenemos en cuenta las elevadas proporciones de casas pequeñas construidas de adobe y con pisos de tierra, fue cierto también que, como en otras partes del país, en ocasiones los precios de los alquileres no se correspondían con niveles adecuados de habitabilidad. Finalmente, si bien los datos censales muestran que rentar una habitación en viviendas de seis o más piezas era frecuente, revelando así la presencia de ciertos conventillos, ${ }^{41}$ la mayor parte de los alquileres se concentraba en unidades con pocas habitaciones (de una a cinco piezas) (véase cuadro 11).

A pesar de la falta de información por ciudad en los censos analizados, pero basándonos en el análisis de todos los datos cuantitativos presentados, inferimos que fue en San Salvador de Jujuy donde la insuficiencia habitacional se manifestó con mayor rigor. En las ciudades de San Pedro y La Quiaca, por su parte, si bien las dificultades habitaciona-

${ }^{40}$ Los periódicos de la época describen a ambas urbes como grandes "ciudades comerciales", donde a lo largo de la década de 1930 comienzan a instalarse cada vez más oficinas estatales y a aumentar el personal público. BPJ-SH, Crónica, 28 de junio de 1935 y 18 de diciembre de 1943.

${ }^{41}$ Conventillo fue el nombre dado en Argentina a aquellos viejos caserones, cuyas habitaciones eran alquiladas por numerosas familias a principios del siglo XX. 





les estuvieron presentes, posiblemente las mayores carencias materiales se concentraron en otras áreas. ${ }^{42}$

En definitiva, y reforzando el supuesto citado en el apartado anterior, en la ciudad capital es muy probable que no sólo los conventillos albergaran núcleos familiares, sino también varias casas particulares. $\mathrm{Mu}$ chos de estos cuartos eran alquilados con el objetivo de contribuir con esta renta a distintos fines, tales como equilibrar el presupuesto familiar, ampliar, mejorar o terminar completamente la propia vivienda. Precisamente el cuadro 11 da cuenta de los múltiples casos en que las familias compartían la vivienda, más allá de que por lo común contara con pocas habitaciones.

\section{REFLEXIONES FINALES}

El crecimiento demográfico que experimentó la provincia de Jujuy en las primeras décadas del siglo XX repercutió indudablemente en la forma de habitar de su población. Los datos censales analizados revelan cómo aquella evolución estuvo acompañada de un notorio aumento de las construcciones de material y de madera en los valles centrales y en los valles subtropicales, respectivamente. Pero en general en Jujuy, así como en gran parte del Noroeste, el uso del adobe en las construcciones constituía una práctica aún muy frecuente de sus habitantes, sobre todo de las comunidades campesinas de ascendencia aborigen de la Quebrada y Puna. Incluso los medios de comunicación, y en ocasiones el gobierno provincial, fomentaban su pervivencia, resaltando el aporte que las casas de adobe brindaban al paisaje norteño, al patrimonio cultural y al incipiente turismo de la provincia. ${ }^{43}$ Por todo ello, al indagar acerca de la diversidad de los materiales empleados en las edificaciones de la provincia, además de factores demográficos y económicos, debe resaltarse asimismo la influencia que tuvieron otros aspectos, como aquellos de índole cultural. Con todo, y pese a estos matices, la mayoría de los hogares jujeños no pareció exhibir grandes adelantos en las condiciones de habitar durante este periodo.

${ }^{42}$ Tanto en San Pedro como en La Quiaca los mayores reclamos presentes en los medios escritos de la época giraron en torno a la necesidad de trabajos en infraestructura urbana (como por ejemplo, la ampliación de los servicios de agua potable y luz eléctrica) más que en el área habitacional. Asimismo, en la ciudad puneña, como en su región, las deficientes condiciones en el campo de la salud y la educación fueron también preocupantes. Estaba claro que el crecimiento de estas ciudades, en niveles notoriamente inferiores al de la urbe capitalina, requería en primer lugar una adecuada satisfacción a estas necesidades.

${ }_{43}$ "[La casa de adobe] armoniza con nuestro paisaje, nuestra historia, nuestro clima y nuestra tradición patricia”, en BPJ-SH, La Opinión, 19 de febrero de 1944, p. 4. 
Tanto los altos índices de hacinamiento como de inquilinos que mostraba Jujuy hacia la mitad del siglo XX confirman las dificultades habitacionales que padecían muchos de sus habitantes. Si bien los elevados niveles de hacinamiento individual en la provincia fueron similares al resto de sus homónimas del Noroeste y diferentes a los de la ciudad de Buenos Aires, ${ }^{44}$ compartía con esta última los más bajos porcentajes de propietarios del país. Las explicaciones de este fenómeno son diversas en el interior de Jujuy. En los valles subtropicales estas proporciones dejan ver cómo la expansión económica experimentada por la zona azucarera, durante este periodo, no halló correlación con mejoras significativas en las formas de habitar de la mayoría de su población, deficiencia, por otro lado, similar a la que exhibían otras dinámicas regiones del país. ${ }^{45}$ En las tierras altas de la Puna revelan la pervivencia de grandes latifundios que provocaba continuas y crecientes protestas, que caracterizaron este periodo, por parte de los campesinos arrendatarios. Todo ello demuestra cómo las problemáticas vinculadas a la vivienda, compartidas en el ámbito nacional, adquirían indudablemente rasgos particulares en el interior del país.

$\mathrm{Al}$ evaluar la deficiencia habitacional, en términos generales, la misma afectó mucho más a los valles centrales, sobre todo al departamento Capital. Dentro de este distrito, fue San Salvador de Jujuy el principal núcleo urbano donde el problema se tornó acuciante. Allí, si bien las viviendas de material habían aumentado, evidentemente el ritmo de crecimiento poblacional fue muy superior. Esto se reflejó, en buena medida, en el poblamiento de la periferia de la ciudad, donde comenzaron a levantarse algunas viviendas medianamente confortables junto a numerosos "ranchos" (casas precarias con rasgos predominantemente rurales). ${ }^{46}$ Asimismo, para muchos el fenómeno se tradujo en la práctica habitual de rentar una habitación para convivir junto a sus familiares. ${ }^{47} \mathrm{El}$ nivel que había adquirido esta cuestión llamó muy pronto la atención de las autoridades guberna-

${ }^{44}$ Buenos Aires mostraba los índices de hacinamiento colectivo más altos del país y los más bajos de hacinamiento individual. Ballent, "Casa", 2000, t. 3.

${ }^{45}$ Como por ejemplo, la provincia de Mendoza, donde la fuerte presencia de viviendas de adobe en las zonas vitivinícolas, según Cerdá, evidencia que el crecimiento económico de la provincia y el proceso de modernización no mostraron similares ritmos. Aunque cabe señalar que el autor hace hincapié principalmente en los hogares construidos de aquel material más que en las grandes dificultades que provocaba el hacinamiento y los inquilinatos como sucedía en Jujuy. Cerdá, "Características", 2007-2008.

${ }_{46}^{46}$ Nicolini y Silva, San, 1973.

${ }^{47}$ La importante presencia de los inquilinos en la ciudad resulta patente en múltiples noticias vinculadas a los alquileres. En 1935 un diario local sostenía: "Los alquileres han vuelto a colocarse a un nivel que no está de acuerdo con el estándar medio de los habitantes de Jujuy. Hay escasez de viviendas y los que especulan con la locación de propiedades, imponen un precio que no es el que debe armonizar con la capacidad económica del núcleo social”, en BPJ-SH, Crónica, 10 de agosto de 1935 , p. 1. 
mentales. ${ }^{48}$ En la década de 1930 radicales y conservadores elaboraron distintas propuestas para enfrentar esta problemática, aunque con escasos resultados ${ }^{49}$ En estos años la prensa también hacía eco denunciando que "cerca de la mitad de obreros y empleados modestos de la Capital ocupan una sola pieza".${ }^{50}$ Con el advenimiento del peronismo las flamantes autoridades tempranamente señalaban la escasez de viviendas como uno de los más graves problemas a resolver en la provincia. ${ }^{51}$

En suma, resulta evidente que, de acuerdo con las características de la vivienda en Jujuy, compartidas en buena parte de la región, las formas de habitar presentaban rasgos muy particulares en relación con otras provincias, principalmente del Litoral. Estos matices resaltan aún más en el área urbana, especialmente en San Salvador de Jujuy, donde uno de los efectos más visibles de la insuficiencia habitacional fue la habitual práctica de muchas familias de compartir la vivienda.$^{52} \mathrm{En}$ tal sentido, si bien en la provincia la proporción de casas con una habitación era alta, su presencia quizá fue más importante en la zona rural. Así, a esta urbe corresponderían en buena medida los altos porcentajes censales de unidades con más de una habitación que eran alquiladas. En este marco, si el modelo nuclear de familia hacia 1936 era predominante en la ciudad de Buenos Aires, fue claro que el mismo difícilmente podría reproducirse por entonces en la ciudad capital jujeña.

\section{Fuentes CONSULTADAS}

\section{Archivos}

AGN Archivo General de la Nación, Buenos Aires.

AHMJ Archivo Histórico Municipal de San Salvador de Jujuy, Jujuy.

${ }^{48}$ A diferencia del caso cuyano donde Cerdá concluye que en Mendoza el problema de la vivienda "no aparece en el discurso de las fuerzas políticas opositoras, en discusiones parlamentarias o en los diarios locales de la época". Lo contrario ocurre en otras provincias como Buenos Aires o Tucumán donde según Natera Rivas la cuestión de los inquilinatos no pasó inadvertida por las autoridades provinciales. Cerdá, "Características", 2007-2008, p. 68, y Natera, "Inquilinatos", 1996.

${ }^{49} \mathrm{El}$ proyecto más importante fue el de la construcción de un barrio de casas baratas en la ciudad capital bajo el gobierno del radical Miguel Tanco en 1930, iniciativa a la postre postergada por el golpe militar de aquel mismo año. AHMJ, La Vanguardia, 9 de mayo de 1930.

${ }^{50}$ BPJ-SH, Crónica, 11 de diciembre de 1936, p. 5.

${ }^{51}$ En un periódico local, el ministro de Gobierno, José H. Martiarena, a poco tiempo de su designación, manifestaba que trabajaría desde su ministerio en concordancia con el gobernador Iturbe "para solucionar los tres grandes problemas de Jujuy: la sanidad, la educación y la vivienda", en BPJ-SH, Jujuy, 24 de febrero de 1947, p. 18.

${ }_{52}$ Es dable suponer que esta experiencia no sólo la padecían las familias que residían en inquilinatos, sino que contemplaba diversas situaciones, como aquellas en las que dentro de la propia casa se alojaba a parientes. 
BPJ-SH Biblioteca Popular de Jujuy, sección Hemeroteca, San Salvador de Jujuy, Jujuy. DIPEC Dirección Provincial de Estadísticas y Censos, San Salvador de Jujuy, Jujuy.

\section{Hemerografia}

Crónica, Jujuy, 1935-1936.

La Vanguardia, Jujuy, 1930.

La Opinión, Jujuy, 1934 y 1944.

Jujuy, Jujuy, 1947.

\section{Bibliografía}

Авоч, Rosa, Viviendas para el pueblo. Espacio urbano y sociabilidad en el barrio de Los Perales. 1946-1955, Buenos Aires, Fondo de Cultura Económica, 2005.

"Arquitectura de la vida doméstica. Familia y vivienda en Buenos Aires, 1914-1960", Anuario IEHS, dossier La historia de la familia, Instituto de Estudios Histórico-Sociales Prof. Juan Carlos Grosso-Universidad Nacional del Centro de la Provincia de Buenos Aires, núm. 23, 2008, Tandil.

BALlENT, ANAHÍ, "La casa para todos: grandeza y miseria de la vivienda masiva" en Fernando Devoto y Marta Madero (dir.), Historia de la vida privada en la Argentina. La Argentina entre multitudes y soledades. De los años treinta a la actualidad, Buenos Aires, Taurus, 2000, t. 3.

"Perón en la ciudad sin esperanza. La política y las políticas urbanas en Buenos Aires" en Patricia Berrotarán, Aníbal Jáuregui y Marcelo Rougier (comps.), Sueños de bienestar en la nueva Argentina. Estado y políticas públicas durante el peronismo, 1946-1955, Buenos Aires, Imago Mundi, 2004.

Las huellas de la política. Vivienda, ciudad, peronismo en Buenos Aires. 1943-1955, Buenos Aires, Universidad Nacional de Quilmes, 2005.

Belli, Elena, Ricardo Slavutsky y Pantaleón Rueda (ed.), Malón de la paz, una historia, un camino, Buenos Aires, Instituto Interdisciplinario Tilcara-Universidad de Buenos Aires, 2007.

BoledA, MARIO, La población del noroeste argentino. Historia y actualidad, Buenos Aires, Legasa/Grupo de Estudios Socio-Demográficos, 1993.

Bolsi, Alfredo (dir.), Problemas poblacionales del noroeste argentino (contribuciones para su inventario), Tucumán, Instituto de Estudios Geográficos Guillermo Rohmeder-Universidad Nacional de Tucumán, 1997.

"Ruralía, tradicionalismo y población en la Puna de Jujuy durante el siglo XX", Mundo Agrario. Revista de Estudios Rurales, Universidad Nacional de La Plata, núm. 10, 2005. 
Cerdá, Juan Manuel, "Características de la vivienda mendocina en la primera mitad del siglo xx", Población y Sociedad, Instituto Superior de Estudios Sociales, núms. 14-15, 2007-2008, Tucumán, pp. 33-70.

Fleitas, María Silvia y Ana Teruel, "Política y movilización campesina en el norte argentino. La cuestión de la tierra indígena en el proceso de ampliación de la democracia", Revista Andina, Centro Bartolomé de las Casas, 2007, Perú.

Germani, Gino, Estructura social de la Argentina, Buenos Aires, Raigal, 1955.

GONZÁLEZ LEANDRI, RicARDO, "La nueva identidad de los sectores populares” en Alejandro Cattaruzza (dir.), Nueva historia argentina. Crisis económica, avance del Estado e incertidumbre politica (1930-1943), Buenos Aires, Sudamericana, 2001.

Kindgard, Adriana, "Tradición y conflicto social en los Andes argentinos: en torno al Malón de la Paz de 1946", Estudios Interdisciplinarios de América Latina y El Caribe (EIAL), School of History-Universidad de Tel Aviv, vol. 15, núm. $1,2004$.

Korn, Francis y Lidia DE LA TORRe, "La vivienda en Buenos Aires 1884-1914", Desarrollo Económico, Instituto de Desarrollo Económico y Social, núm. 98, vol. 25 julio-septiembre de 1985, Buenos Aires.

LeCOUnA, Diego, Legislación sobre locaciones urbanas y el problema de la vivienda, Buenos Aires, Consejo Empresarial de América Latina, 1992, t. 2.

LIERnUR, Jorge F., "Casas y jardines. La construcción del dispositivo doméstico moderno (1870-1930)" en Fernando Devoto y Marta Madero (dir.), Historia de la vida privada en la Argentina. La Argentina plural (1870-1930), Buenos Aires, Taurus, 2000.

Nari, Marcela, Políticas de maternidad y maternalismo político. Buenos Aires (18901940), Buenos Aires, Biblos, 2004.

Natera Rivas, Juan José, "Inquilinatos en la ciudad de San Miguel de Tucumán en la primera mitad del siglo XX", Población y Sociedad, Instituto Superior de Estudios Sociales, núm. 4, diciembre de 1996, Tucumán, pp. 178-200.

Nicolini, Alberto y Marta Silva, San Salvador de Jujuy, 1561-1961. Desarrollo urbano y arquitectónico, Tucumán, Universidad Nacional de Tucumán, 1973.

Ortiz de D'ARterio, J. PATricia y MARtha E. CAillou, "Una primera aproximación al estudio del crecimiento natural (1910-1992)" en AlFREDo BoLSI (dir.), Problemas poblacionales del noroeste argentino (contribuciones para su inventario), Tucumán, Instituto de Estudios Geográficos Guillermo Rohmeder-Universidad Nacional de Tucumán, 1997.

Otero, Hernán, "Demografía política e ideológica estadística en la estadística censal argentina, 1969-1914”, Anuario IEHS, Instituto de Estudios HistóricoSociales Prof. Juan Carlos Grosso-Universidad Nacional del Centro de la Provincia de Buenos Aires, núm. 14, 1999, Tandil. 
"Censos antiguos: 1869, 1895, 1914, 1947" en Susana Torrado (comp.), Población y bienestar en la Argentina del primero al segundo centenario. Una historia social del siglo XX, Buenos Aires, EDHASA, 2007, t. 1.

"El concepto de población en el sistema estadístico nacional" en SUSANa Torrado (comp.), Población y bienestar en la Argentina del primero al segundo centenario. Una historia social del siglo XX, Buenos Aires, EDHASA, 2007, t. 1.

Rigotti, Ana María, "La ciudad y la vivienda como ámbitos de la política y la práctica profesional" en RICARDO FALCón (dir.), Nueva historia argentina. Democracia, conflicto social y renovación de ideas (1916-1930), Buenos Aires, Sudamericana, 2000.

RouQuiÉ, Alain, Extremo Occidente. Introducción a América Latina, Buenos Aires, Emecé, 1991.

SAla, Gabriela, "Redistribución espacial y procesos migratorios" en DANIEL SANTAMARÍA (comp.), Jujuy: arqueología, historia, economía, sociedad, Jujuy, CEIC/ Ediciones Cuadernos del Duende, 2005.

Sierra e Iglesias, Jobino, Un tiempo que se fue. Vida y obra de los hermanos Leach, Jujuy, Ediunju, 1998.

Teruel, ANA, "Panorama económico y socio-demográfico en la larga duración (siglos XiX y Xx)" en Ana Teruel y Marcelo Lagos (dir.), Jujuy en la historia. De la colonia al siglo XX, Jujuy, Ediunju, 2006.

y María TERESA Bovi, "El ordenamiento de la propiedad territorial en Jujuy. Del antiguo régimen a la modernidad" en Ana Teruel (dir.), Problemas nacionales en escalas locales. Instituciones, actores y prácticas de la modernidad en Jujuy, Rosario, Prohistoria, 2010.

Torrado, Susana, "Transición de la familia: tamaño y morfología" en Susana TORrado (comp.), Población y bienestar en la Argentina del primero al segundo centenario. Una historia social del siglo XX, Buenos Aires, EDHASA, 2007, t. II.

TORRe, JUAN CARLOS y Elisa PASTORIZA, "La democratización del bienestar" en JuAN CARlos TORRe (dir.), Nueva historia argentina. Los años peronistas (19461955), Buenos Aires, Sudamericana, 2002.

TORres, Horacio, El mapa social de Buenos Aires (1940-1990), Buenos Aires, FAdU/ Universidad de Buenos Aires, 1993.

Yujnovsky, Oscar, "Políticas de vivienda en la ciudad de Buenos Aires", Desarrollo Económico, Instituto de Desarrollo Económico y Social, vol. 14, núm. 54, julio-septiembre de 1974, Buenos Aires. 